\title{
FURTHER GENERALIZATIONS OF NEUMANN'S INTEGRAL
}

\author{
W. PYE \\ (Received 25 November 1970; revised 1 April 1971) \\ Communicated by B. Mond
}

\begin{abstract}
1. Abstract
A generalization of Neumann's integral connecting the two kinds of Legendre function is obtained. It contains an extra parameter which is not a function of the parameters of the Legendre functions, unlike all previous extensions of the original formula. These extensions are shown to be particular cases of the new generalization and some further particular cases are also indicated.
\end{abstract}

\section{Introduction}

In 1848 Neumann ([1]; page 292) expressed the Legendre function of the second kind in terms of that of the first kind:

$$
Q_{n}(z)=\frac{1}{2} \int_{-1}^{1} \frac{P_{n}(t)}{z-t} d t
$$

for all complex $z$ except those real values in the interval $[-1,1]$ and for $n$ a non-negative integer. Wrinch [7] obtained, in 1930, a generalization which inserted a polynomial of degree not greater than $n$, namely,

$$
Q_{n}(z) p(z)=\frac{1}{2} \int_{-1}^{1} \frac{P_{n}(t)}{z-t} p(t) d t
$$

Since then, further generalizations have been given by Gormley [3] in 1934, Robin [5] in 1957 and Love [4] in 1965. Gormley and Robin extended (2) to the associated Legendre functions but still one of the parameters was required to be integral. Love obtained results for both the associated and "unassociated" functions which removed this restriction. These results were for functions of the type

$$
E(z)=(z+1)^{x}(z-1)^{\beta} Q_{v}^{u}(z)
$$

where $\alpha$ and $\beta$ are functions of $v$ and $\mu$, with sometimes a Wrinch-type generalization being added. 
This paper gives generalizations of Neumann's integral which free one of the parameters $\alpha$ and $\beta$ from the restriction that it should be a function of $v$ and $\mu$. It will also be shown that the results cited above are special cases of this more general result. A further special case is also indicated.

The main result obtained is:

(4) If $\mathrm{re}(1+v)>0$, min $\operatorname{re}\left(\beta \pm \frac{1}{2} \mu+1\right)>0, v+\mu$ is not an integer, $z$ is in the plane cut along the real axis from -1 to $+1, k$ is any integer satisfying

$$
0 \leqq k<\min \operatorname{re}\left(v-\beta \pm \frac{1}{2} \mu+1\right)
$$

and $p(z)$ is any polynomial of degree $k$ or less, then

$$
\begin{aligned}
& (z+1)^{\nu-\beta-k}(z-1)^{\beta} Q_{\nu}^{u}(z) p(z) \\
& =\frac{e^{i \mu \pi}}{2 \sin (v+\mu) \pi} \int_{-1}^{1}\left\{\frac{(1+t)^{\nu-\beta-k}(1-t)^{\beta} \sin \left(v-\beta+\frac{1}{2} \mu\right) \pi}{z-t} p(t)\right. \\
& \left.+\frac{(1-t)^{\nu-\beta-k}(1+t)^{\beta} \sin \left(\beta+\frac{1}{2} \mu\right) \pi}{z+t} p(-t)\right\} P_{\nu}^{\prime \prime}(t) d t
\end{aligned}
$$

The case $\beta=0$ appears to be a result on the same level of generality as Love's, but new.

The term "cut plane" throughout this paper will refer to the complex plane omitting the closed interval $[-1,1]$ of the real axis. All powers occurring are principal values, that is, $z^{w}$ is defined to be $\exp (w \log z)$ where $-\pi<\operatorname{im}(\log z) \leqq \pi$.

\section{Regularity of $E(z)$}

To establish these results we use Cauchy's integral, the usual method of establishing Neumann's integral (see, for instance, [1] p. 292 or [2] p. 153), rather than employing successive integrations by parts and orthogonality relations as the later workers have done. We find that

(6) E(z) is regular in the cut plane if $\mathrm{re}(1+v)>0, v+\mu$ is not an integer, and $\alpha=v-r-\beta$.

Proof. In the cut plane $E(z)$ has, in general, a branch point at infinity. However, by [2]: equation 3.2(5), E(z) behaves near infinity as a multiple of $z^{\alpha+\beta-v-1}$. So that in the case where $\alpha+\beta-v$ is an integer there is no branch point at infinity. Thus $E(z)$ can be defined in some neighbourhood of the segment of the real axis from 1 to infinity in terms of principal values. Analytic continuation then gives the required result. 


\section{Application of Cauchy's Integral.}

The above result enables Cauchy's integral to be used on the contour $C$ shown in the diagram. The radii of the circles $C_{1}, C_{2}$ and $C_{4}$ are $R, \varepsilon$ and $\eta$

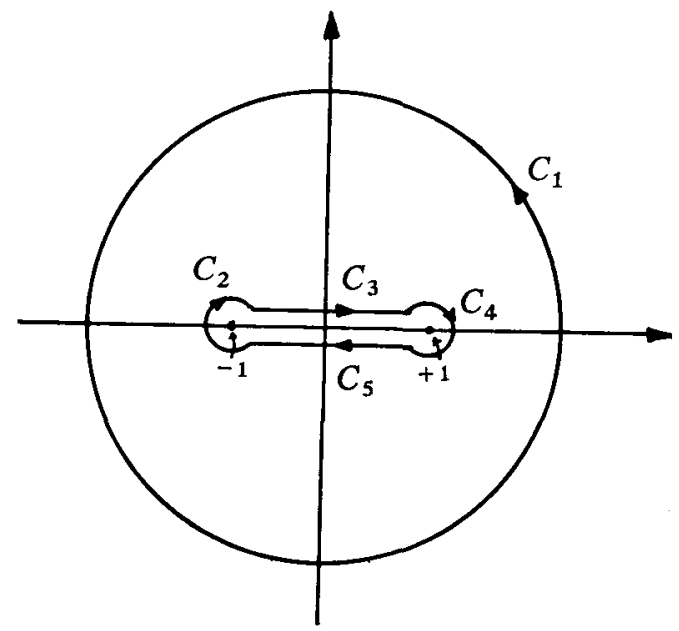

respectively and values of these are chosen so that the outer circle is large enough and the two straight line segments and small circles close enough to the branch cut to ensure that $z$ lies inside the contour. So

$$
\frac{1}{2 \pi i} \oint_{c} \frac{E(w)}{w-z} d w=I_{1}+I_{2}+I_{3}+I_{4}+I_{5}
$$

where $I_{r}=\frac{1}{2 \pi i} \int_{C r} \frac{E(w)}{w-z} d w$.

On $C_{1}, w=R e^{i \theta}$ and so by ([2]: equation $\left.3.2(36)\right) I_{1}$ is a multiple, independent of $z$ and $R$, of

$$
\begin{gathered}
\int_{0}^{2 \pi} \frac{R e^{i \theta}}{R e^{i \theta}-z}\left(R e^{i \theta}+1\right)^{\alpha+\frac{1}{2} \mu-v-1}\left(R e^{i \theta}-1\right)^{\beta-\frac{1}{2} \mu} F \\
\\
\left(1+v-\mu, 1+v ; 2+2 v ; \frac{2}{1+R e^{i \theta}}\right) d \theta
\end{gathered}
$$

Provided $R$ is sufficiently large,

$$
\left|I_{1}\right| \leqq K_{1} \frac{R}{R-|z|}(R \pm 1)^{r e\left(\alpha+\frac{1}{2} \mu-v-1\right)}(R \pm 1)^{r e\left(B-\frac{1}{2} \mu\right)}
$$

where $K_{1}$ is a positive constant, and the upper or lower sign is taken according as re $\left(\alpha+\frac{1}{2} \mu-v-1\right)$ or $\operatorname{re}\left(\beta-\frac{1}{2} \mu\right)$ is positive or negative. Thus as $R \rightarrow \infty, I_{1} \rightarrow 0$ if $\operatorname{re}(\alpha+\beta-v-1)<0$. 
On $C_{2}, w=-1+\varepsilon e^{i \theta}$ and so by ([2]: equation $\left.3.2(33)\right) I_{2}$ is equal to

$$
\begin{aligned}
& \int_{0}^{2 \pi} \frac{\varepsilon e^{i \theta}}{-1+\varepsilon e^{i \theta}-z}\left\{\left(\varepsilon e^{i \theta}\right)^{\alpha+\frac{1}{2} \mu}\left(-2+\varepsilon e^{i \theta}\right)^{\beta-\frac{1}{2} \mu} A F_{1}\right. \\
&+\left(\varepsilon e^{i \theta}\right)^{\alpha-\frac{1}{2} \mu}\left(-2+\varepsilon e^{i \theta \beta+\frac{1}{2} \mu} B F_{2}\right\} d \theta
\end{aligned}
$$

where $F_{1}$ and $F_{2}$ are the hypergeometric functions $F\left(-v, 1+v ; 1+\mu ; \frac{1}{2} \varepsilon e^{i 0}\right)$ and $F\left(-v, 1+v ; 1+\mu ; \frac{1}{2} \varepsilon e\right)^{i \theta}$ and $A$ and $B$ are constants, independent $\varepsilon$ and $z$.

For this result we impose the restrictions that $\nu+\mu$ is not a negative integer and that $\mu$ is not an integer; the latter restriction will removed later.

Provided $\varepsilon$ is sufficiently small,

$$
\left|I_{2}\right| \leqq \frac{\varepsilon}{|z+1|-\varepsilon}\left\{K_{2} \varepsilon^{r e\left(\alpha+\frac{1}{2} u\right)}(2 \pm \varepsilon)^{r e\left(\beta-\frac{1}{2} u\right)}+K_{3} \varepsilon^{r e\left(z-\frac{1}{2} \mu\right)}(2 \pm \varepsilon)^{r\left(\beta+\frac{1}{2} u\right)}\right\}
$$

where $K_{2}$ and $K_{3}$ are positive constants and the upper or lower sign is taken in the first term according as $\operatorname{re}\left(\beta-\frac{1}{2} \mu\right)$ is positive or negative and in the second term according as $\operatorname{re}\left(\beta+\frac{1}{2} \mu\right)$ is positive or negative. Thus as $\varepsilon \rightarrow 0, I_{2} \rightarrow 0$ if $\operatorname{re}\left(\alpha+\frac{1}{2} u+1\right)>0$ and $\operatorname{re}\left(\alpha-\frac{1}{2} \mu+1\right)>0$.

On $C_{4}, w=1+\eta e^{i \theta}$ and a similar treatment using ([2]: equation 3.2(32)) indicates that as $\eta \rightarrow 0, I_{4} \rightarrow 0$ if $v+\mu$ is not a negative integer, $\mu$ is not an integer, $\operatorname{re}\left(\beta+\frac{1}{2} \mu+1\right)>0$ and $\operatorname{re}\left(\beta-\frac{1}{2} \mu+1\right)>0$.

Thus, using Cauchy's integral and the above results, we have

$$
\begin{aligned}
& (z+1)^{\alpha}(z-1)^{\beta} Q_{v}^{u}(z) \\
& =\frac{1}{2 \pi i} \int_{-1}^{1} \frac{(1+t)^{\alpha}(1-t)^{\beta}}{z-t}\left\{e^{-i \beta \pi} Q_{v}^{u}(t-0 i)-e^{i \beta \pi} Q_{v}^{u}(t+0 i)\right\} d t
\end{aligned}
$$

Use of Erdélyi ([2]: equation $3.4(9)$ ) gives, for $-1<t<1$,

$$
\begin{aligned}
& Q_{v}^{\mu}(t-0 i)=e^{\frac{1}{2} i \mu \pi}\left[Q_{v}^{u}(t)+\frac{1}{2} i \pi P_{v}^{u}(t)\right] \quad \text { and } \\
& Q_{v}^{\mu}(t+0 i)=e^{i i \mu \pi}\left[Q_{v}^{u}(t)-\frac{1}{2} i \pi P_{v}^{\mu}(t)\right] .
\end{aligned}
$$

So, using Erdélyi ([2]: equation 3.4 (14)), which can be shown to hold in $-1<t<1$, and supposing that $v+\mu$ is not an integer,

$$
\begin{gathered}
e^{-i \beta \pi} Q_{v}^{\mu}(t-0 i)-e^{i \beta \pi} Q_{v}^{\mu}(t+0 i) \\
=\pi i e^{i \mu \pi}\left\{P_{v}^{\mu}(t) \cos \left(\beta+\frac{1}{2} \mu\right) \pi-\frac{2 \sin \left(\beta+\frac{1}{2} \mu\right) \pi}{\pi} Q_{v}^{\mu}(t)\right\} \\
=\frac{\pi i e^{i \mu \pi}}{\sin (v+\mu) \pi}\left\{P_{v}^{\mu}(t) \sin \left(v-\beta+\frac{1}{2} \mu\right) \pi+P_{v}^{\mu}(-t) \sin \left(\beta+\frac{1}{2} \mu\right) \pi\right\} .
\end{gathered}
$$


Thus (7) becomes

$$
\begin{gathered}
(z+1)^{\alpha}(z-1)^{\beta} Q_{v}^{\mu}(z)=\frac{e^{i \mu \pi}}{2 \sin (v+\mu) \pi} \int_{-1}^{1} \frac{(1+t)^{\alpha}(1-t)^{\beta}}{z-t}\left\{P_{v}^{\mu}(t) \sin \left(v-\beta+\frac{1}{2} \mu\right) \frac{1}{2} \pi\right. \\
\left.+P_{v}^{\mu}(-t) \sin \left(\beta+\frac{1}{2} \mu\right) \pi\right\} d t
\end{gathered}
$$

The two terms may be integrated separately, since the resulting integrals are both convergent. This may be seen by reference to ([4]: page 449). If $t$ is replaced by $-t$ in the second integral and $\alpha$ is replaced by $v-r-\beta$ in both integrals, as required by (6), we find that:

(8) If $\mathrm{re}(v+1)>0, r$ is a non-negative integer, $r<\min \operatorname{re}\left(v-\beta \pm \frac{1}{2} \mu+1\right)$, $\min \operatorname{re}\left(\beta \pm \frac{1}{2} \mu+1\right)>0, v+\mu$ is not an integer and $\mu$ is not an integer, then

$$
\begin{gathered}
(z+1)^{v-r-\beta}(z-1)^{\beta} Q_{v}^{u}(z) \\
=\frac{e^{i \mu \pi}}{2 \sin (v+\mu) \pi} \int_{-1}^{1}\left\{\frac{(1+t)^{v-r-\beta}(1-t)^{\beta} \sin \left(v-\beta+\frac{1}{2} \mu\right) \pi}{z-t}\right. \\
\left.+\frac{(1-t)^{v-r-\beta}(1+t)^{\beta} \sin \left(\beta+\frac{1}{2} \mu\right) \pi}{z+t}\right\} P_{v}^{u}(t) d t
\end{gathered}
$$

Both sides of (9) are analytic functions of $\mu$ which are regular at integer values of $v$ so long as $v+\mu$ is not an integer. Hence (9) can be extended to integral values of $\mu$ by analytic continuation.

\section{Wrinch-Type Generalization.}

Taking linear combinations of the result (9) for various admissible values of $r$ and changing the order of summation and integration yields

$$
\begin{aligned}
& (z+1)^{v-\beta}(z-1)^{\beta} Q_{v}^{\mu}(z) \sum_{r=0}^{k} \frac{a_{r}}{(1+t)^{r}} \\
& =\frac{e^{i \mu \pi}}{2 \sin (v+\mu) \pi} \int_{-1}^{1}\left\{\frac{(1+t)^{\nu-\grave{p}}(1-t)^{\beta} \sin \left(v-\beta+\frac{1}{2} \mu\right) \pi}{z-t} \sum_{r=0}^{k} \frac{a_{r}}{(1+t)^{r}}\right. \\
& \left.+\frac{(1-t)^{\nu-\beta}(1+t)^{\beta} \sin \left(\beta+\frac{1}{2} \mu\right) \pi}{z+t} \sum_{r=0}^{k} \frac{a_{r}}{(1-t)^{r}}\right\} P_{v}^{\mu}(t) d t
\end{aligned}
$$

which simplifies to the result (5) under the conditions (4). 


\section{Special Cases.}

1. Putting $\beta=-\frac{1}{2} \mu$ in (5) and (4) yields a result almost the same as one found by Love ([4]: equation (36)), from which it differs in two respects-the restriction that $v+\mu$ should not be integral and the values of $z$ for which it holds. The restriction can be removed by proceeding directly to the result, rather than through the general result.

The result holds for all $z$ except those in the interval $[-1,1]$, whereas Love's results exclude all values in $(-\infty, 1]$. This arises because Love's approach considers each factor of $E(z)$ separately as an analytic function while the methods adopted in this work consider $E(z)$ as a single function allowing the branch cut along $(-\infty,-1)$ to be dispensed with.

If we allow $v+\mu$ to be integral and put $p(z)=1$, we obtain Gormley's result ([3]: equation (4)). The conditions imposed also simplify to those of Gormley.

2. When $\beta=+\frac{1}{2} \mu$ another of Love's results ([4]: equation (45)) is found under exactly the same conditions but for the extended set of values of $z$.

3. The substitution of $v+\frac{1}{2} \mu$ for $\beta$ requires $k$ to be zero so that $p(z)=1$.

Replacement of $z$ by $-z$ and $Q_{v}^{\mu}(-z)$ by $-e^{ \pm i v \pi} Q_{v}^{u}(z)$ then yields a generalization of Robin's result ([6]: equation (187)). The same result was found by Love ([4]: equation (30)), but for the more restricted set of values of $z$ indicated earlier.

4. When $\beta=0$ a result is found which does not seem to appear elsewhere in the literature. However, the subcase with $\mu=0$ has been given by Love ([4]: equation (3)).

5. The case with $\beta=v$ is analogous to the result for which $\beta=0$ but with the factor $(z-1)^{\nu}$ instead of $(z+1)^{\nu}$. Some generality is lost however since the integer $k$ becomes zero and $p(z)=1$.

6. Substitution for $\beta$ of $\frac{1}{2}(v-\mu)$ and $\frac{1}{2}(v+\mu)$ yield results analogous to those for which $\beta$ is $-\frac{1}{2} \mu$ and $v+\frac{1}{2} \mu$ respectively but with the factor $\left(z^{2}-1\right)^{\frac{1}{2} v}$ instead of $(z+1)^{v}$ or $(z-1)^{v}$. The analogy is not however a strict one since the integrals contain two terms rather than the single terms of the earlier cases.

The latter result has more generality than its analogue since $k$ is not necessarily zero and the polynomial $p(z)$ can consequently be retained.

Finally, I express my appreciation to Professor E. R. Love for several valuable suggestions and to the referee for his helpful comments concerning the presentation of this paper. 


\section{References}

[1] E. T. Copson, Theory of Functions of a Complex Variable (Oxford, 1935).

[2] A. Erdélyi, W. Magnus, F. Oberhettinger and F. Tricomi, Higher Transcendental Functions, Vol. I (McGraw-Hill, New York, 1953).

[3] P. G. Gormley, 'A Generalization of Neumann's Formula for $\mathrm{Q}_{n}$ (z)', J. London Math. Soc. 9 (1934), 149-152.

[4] E. R. Love, 'Franz Neumann's Integral of 1848', Proc. Camb. Phil. Soc. 61, (1965), 445-456.

[5] L. Robin, Fonctions sphériques de Legendre et fonctions sphériodales, Vol. I (Gauthier-Villars, Paris, 1957).

[6] L. Robin, Fonctions sphériques de Legendre et fonctions sphériodales, Vol. II (Gauthier-Villars, Paris 1958).

[7] Dorothy Wrinch, 'On Some Integrals involving Legendre Polynomials'. Phil. Mag. 7(10) (1930), 1037-1043.

Secondary Teachers' College and University of Melbourne

Victoria, 3052

Australia 